\title{
The Nitro Group as a Masked Electrophile in Covalent Enzyme Inhibition
}

\author{
Sneha Ray ${ }^{\dagger}$, Dale F. Kreitler ${ }^{\ddagger}$, Andrew M Gulick ${ }^{\ddagger}$, and Andrew S Murkin ${ }^{\dagger}{ }^{*}$ \\ † Department of Chemistry, University at Buffalo, Buffalo, New York 14260-3000, United States \\ ‡ Hauptman-Woodward Institute and Department of Structural Biology, University at Buffalo, \\ Buffalo, New York 14203-1102, United States
}

\begin{abstract}
We report the unprecedented reaction between a nitroalkane and an active-site cysteine residue to yield a thiohydroximate adduct. Structural and kinetic evidence suggests the nitro group is activated by conversion to its nitronic acid tautomer within the active site. The nitro group, therefore, shows promise as a masked electrophile in the design of covalent inhibitors targeting binding pockets with appropriately placed cysteine and general acid residues.
\end{abstract}

Recent years have seen renewed interest in the development of inhibitors that function by covalently modifying their targets. To avoid the obvious potential for toxicity resulting from off-target reactions, so-called targeted covalent inhibitors are designed with a weakly electrophilic group that reacts selectively with a nucleophilic residue on the target only upon binding and proper positioning of the reactive functionalities. ${ }^{2-3}$ Novel electrophilic functional groups with low intrinsic reactivity are therefore highly desirable for this rapidly developing inhibitor design strategy.

In the current study, we report the previously unrealized ability of a nitroalkane to form a covalent adduct with a protein without the assistance of cofactors. This discovery stemmed from an investigation of isocitrate lyase (ICL), a drug target essential for the survival of Mycobacterium tuberculosis during the latent stage of infection. Whereas most organisms depend on carbohydrates as a carbon source, M. tuberculosis is capable of subsisting on acetate and fatty acids within the hypoxic confines of macrophages by utilizing the glyoxylate shunt. ${ }^{4}$ As the first of two steps in the shunt, ICL catalyzes the reversible retroaldol cleavage of the tricarboxylic acid cycle intermediate isocitrate into succinate and glyoxylate (Scheme 1a); malate synthase subsequently converts glyoxylate to L-malate, which, together with succinate, is ultimately converted to oxaloacetate for gluconeogenesis. Due to its absence in humans, ICL is a desirable target for inhibitor design.

\footnotetext{
"Corresponding Author amurkin@buffalo.edu.

Notes

The authors declare no competing financial interests.

Supporting Information

Supporting Information Available: This material is available free of charge via the Internet

Experimental details, table of X-ray data collection and refinement statistics, kinetics plots, time-dependent mass spectra
} 
3-Nitropropionate (3-NP), an analogue of succinate, bearing a nitro group in place of one of the carboxylates, is a potent time-dependent inhibitor of ICL. ${ }^{5-6}$ Like other nitroalkanes, 3$\mathrm{NP}\left(\mathrm{p} K_{\mathrm{a}}=9.0^{7}\right)$ exists partially as its conjugate base propionate-3-nitronate $(\mathrm{P} 3 \mathrm{~N})$ in aqueous solution near neutral $\mathrm{pH}$ (Scheme 1b). Taking advantage of the slow rate of proton transfer to/from 3-NP, ${ }^{7}$ Schloss and Cleland reported that the onset of inhibition of Pseudomonas indigofera ICL was accelerated as the inhibitor was converted from the free acid to fully ionized form by increasing the $\mathrm{pH}$ in preincubated samples. ${ }^{5}$ This prompted the authors to conclude that $\mathrm{P} 3 \mathrm{~N}$ is the true form of the inhibitor and that the onset of inhibition is the result of the slow formation of a tight binary complex (Scheme 1c). While our previous studies demonstrated time-dependent inhibition also occurs with the $M$. tuberculosis enzyme, we observed a large inverse solvent isotope effect (i.e., the onset of inhibition was twice as fast in $\mathrm{D}_{2} \mathrm{O}$ ) that cannot be explained by this mechanism. ${ }^{6}$ Additionally puzzling is that after complete inhibition of $P$. indigofera ICL by pre-incubation with 3-NP, jump-dilution into assay mixtures containing excess succinate, which competes with 3 -NP, resulted in recovery of only $25 \%$ of the uninhibited activity. ${ }^{5}$

To address these inconsistencies, we measured inhibition kinetics with $M$. tuberculosis ICL using 3-NP or P3N at pH 7.5. Inhibition by 3-NP, initiated by addition of ICL, was monitored spectrophotometrically in the isocitrate-synthesis direction by following the reduction of $\mathrm{NADP}^{+}$by isocitrate dehydrogenase. Inhibition by $\mathrm{P} 3 \mathrm{~N}$ required rapid mixing of the fully ionized inhibitor (prepared in $0.1 \mathrm{M} \mathrm{NaOH}$ ) and succinate with the rest of the assay components buffered at $\mathrm{pH} 7.5$, in order to minimize the extent of reprotonation back to 3-NP. Reprotonation monitored spectrophotometrically (SI Figure S1) showed that using $10 \mathrm{mM}$ potassium phosphate buffer, $<10 \%$ of the total $\mathrm{P} 3 \mathrm{~N}$ would have been converted to 3 NP by the end of the assay. Both inhibitors demonstrated slow-onset inhibition (Figure 1a and SI Figure S2a), though it was not possible to distinguish whether any residual steadystate rate remained after prolonged incubations. To assess the reversibility of inhibition, we therefore conducted jump-dilution experiments similar to those by Schloss and Cleland. ${ }^{5}$ Consistent with their findings but to a much greater extreme, we observed very limited recovery of activity $(\sim 0.09 \%)$ over the course of $20 \mathrm{~h}$, compared to a control that had not been pre-incubated with inhibitor (Figure S2b and SI Figure S2b).Inclusion of $5 \mathrm{mM}$ DTT had no noticeable effect on the activity.

The unexpected failure of ICL to recover most of its activity led us to hypothesize that it was due to irreversible formation of a covalent complex. To investigate this possibility, we performed electrospray ionization mass spectrometry on $M$. tuberculosis ICL. To our surprise, the protein's mass increased from $48,783 \pm 1$ to $48,884 \pm 1 \mathrm{Da}$ in the presence of 3NP (Figure 1c,d). Although extended incubation periods led to complete modification (SI Figure S3), the mass increase was accelerated by the presence of glyoxylate (Figure 1e), indicative of synergistic binding of the inhibitor. This increase of $101 \pm 1 \mathrm{Da}$ is 18 less than the molecular weight of $3 \mathrm{NP}$, suggesting that covalent modification of ICL may be accompanied by loss of a molecule of water.

Unequivocal identification of the protein modification was obtained by X-ray crystallography. Interestingly, Sharma et al. published the structure of the C191S mutant of M. tuberculosis ICL bound with $\mathrm{Mg}^{2+}$, glyoxylate, and 3-NP, in which no covalent 
modification was observed (Figure 2a). ${ }^{1}$ While no explanation for mutation of the active-site residue was provided, it is possible that the covalent modification of the wild-type enzyme interfered with crystallographic analysis under their conditions. Although the pseudo- $C_{2}$ symmetry of the inhibitor prevents definitive assignment of its orientation in this complex, the depiction in Figure 2a is compatible with formation of a covalent adduct (vide infra). Of note in this structure is the $3.0 \AA$ distance between the hydroxyl group of Ser191 and C-3 of the inhibitor. Additionally, Arg228 and Glu285 make contacts with the oxygen atoms of the nitro group. Glu285 is believed to exist in the acid form in order to serve as a hydrogen-bond donor and to avoid electrostatic repulsion, especially when succinate is bound in place of 3NP. In the presence of $\mathrm{Mg}^{2+}$ and 3-NP, wild-type ICL formed a covalent adduct between the sulfur of Cys191 and C3 (Figure 2b). The trigonal planar geometry at C-3 is consistent with a thiohydroximate with $\mathrm{Z}$ stereochemistry and inconsistent with a nitroso tautomer. This adduct, which lacks one of the oxygen atoms present in the nitro group, agrees with the observed increase in mass. Pyruvate, present endogenously during protein expression, was found coordinated to $\mathrm{Mg}^{2+}$ in place of glyoxylate, as it has been shown to do previously. ${ }^{8}$ Soaking the crystals with glyoxylate resulted in displacement of the bound pyruvate at three of the eight crystallographically independent active sites and a nearly identical structure (PDB 6C4C).

Based on the covalent nature of inhibition, we performed additional analysis of the kinetic data presented earlier (Figure 1a), fitting each curve to an exponential function. Plots of the observed rate constants $\left(k_{\mathrm{obs}}\right)$ vs. [3-NP] or [P3N] were linear (SI Figure S4), with slopes equal to the respective second-order rate constant for inactivation $\left(k_{\text {inact }} / K_{\mathrm{I}}\right)$. While this pattern is consistent with a single-step binding process, it is implausible considering the chemical transformation that must occur. We therefore favor a minimal two-step scheme (Scheme 1d) in which the initial equilibrium is far from saturation over all concentrations of inhibitor tested (i.e., [inhibitor] $\ll<k_{-1} / k_{1}$ ). Global fitting of the data gave $k_{\text {in }}$ act $/ K_{\mathrm{I}}$ values of $270 \pm 50$ and $26,000 \pm 3,000 \mathrm{M}^{-1} \mathrm{~s}^{-1}$, respectively, for 3-NP and $\mathrm{P} 3 \mathrm{~N}$ in $10 \mathrm{mM}$ potassium phosphate ( $\mathrm{pH}$ 7.5). The second-order rate constant for 3-NP is about fivefold higher than that for 2- $C$-vinyl-D-isocitrate, a mechanismbased inactivator that modifies Cys191 via an enzymegenerated Michael acceptor. ${ }^{9}$ The value is also comparable to the value of $1050 \mathrm{M}^{-1}$ $\mathrm{s}^{-1}$ reported for the $P$. indigofera enzyme at $\mathrm{pH} 8,{ }^{5}$ considering there is about threefold more $\mathrm{P} 3 \mathrm{~N}$ present at equilibrium at that $\mathrm{pH}$. That $25 \%$ of the uninhibited activity was recovered in jump-dilution experiments with this enzyme, in contrast to $M$. tuberculosis ICL, may indicate a partitioning of the bound inhibitor between covalent and non-covalent complexes that differs between the orthologues.

In light of the covalent nature of inhibition and the $\sim 100$ fold faster rate of inactivation compared to 3-NP, we propose that $\mathrm{P} 3 \mathrm{~N}$ is an intermediate in the formation of the thiohydroximate adduct. However, considering that the thermodynamic ratio of the two inhibitory species at $\mathrm{pH} 7.5$ is only $~ 30$, it is evident that conversion of 3-NP to P3N during inactivation is partially rate limiting. Consistent with this, we observed catalysis by buffer, both by anionic (HEPES) and cationic (imidazole) buffers (phosphate could not be tested due to its limited solubility in the presence of $\mathrm{Mg}^{2+}$ ). Both buffers exhibited hyperbolic concentration dependence (SI Figure S5), consistent with a transition in rate-limiting step from 3-NP ionization to a subsequent process involved in covalent adduct formation. 
A mechanism is suggested for this unprecedented protein modification, in which $\mathrm{P} 3 \mathrm{~N}$ is an intermediate, formed by deprotonation of 3-NP (Scheme 2). In this scheme, buffer catalyzes deprotonation of 3-NP to yield P3N, which binds to ICL. Although P3N is nucleophilic, protonation of one of the nitronate oxygens, presumably by Glu285, would lead to nitronic acid $\mathbf{1}$, which is electrophilic and readily reacts with the neighboring cysteine residue to yield the initial covalent species $\mathbf{2}$. The sulfhydryl most likely attacks as the thiolate, consistent with the inverse solvent isotope effect we observed previously. ${ }^{6}$ Protonation of the other nitronate oxygen, possibly by Arg228, may occur prior to or following C-S bond formation. Dehydration of $\mathbf{2}$ via either an $\mathrm{E}_{\mathrm{i}}$-type elimination (shown) or stepwise process via a nitroso intermediate then results in formation of the stable thiohydroximate adduct $\mathbf{3}$. This mechanism, which parallels that proposed for reaction of nitroalkanes with thiosilanes, 10 differs greatly from that employed in inhibition of succinate dehydrogenase by 3-NP, which instead uses its flavin cofactor to oxidize the inhibitor to a nitroalkene, which serves as a Michael acceptor. ${ }^{11}$

Although rare, thiohydroximates have been observed biologically as intermediates in the synthesis of some phosphonate natural products ${ }^{12}$ and glucosinolates. ${ }^{13}$ Interestingly, in formation of the latter, a thiohydroximate has also been proposed to result from reaction of a nitronic acid with a thiol nucleophile, presumably glutathione; ${ }^{13}$ however, in this case, the nitronic acid is formed by oxidation of an aldoxime rather than tautomerization of a nitroalkane, ${ }^{14}$ as demonstrated here.

The results of this study bring to question whether nitro groups can function more generally to modify other proteins (enzymes or receptors) by placement in regions of known ligands proximal to (1) an acidic residue and (2) a nucleophilic group (e.g., cysteine). As such, they could function as masked warheads for targeted covalent inhibition. ${ }^{2-3} \mathrm{~A}$ limitation of using nitro compounds as drugs, however, is their potential for toxicity, most commonly via metabolic reduction to reactive nitroso and hydroxylamine derivatives. While this is a valid concern that must be investigated early in the drug discovery process, there are a number of examples that are pharmaceutically active and pose no serious safety risks. ${ }^{15}$

\section{Supplementary Material}

Refer to Web version on PubMed Central for supplementary material.

\section{ACKNOWLEDGMENT}

This work was supported by NSF CAREER Award CHE1255136 (A.S.M.) and NIH Grant GM-116957 (A.M.G.). We thank T. Meek (Texas A\&M) for helpful discussions.

\section{REFERENCES}

(1). Sharma V; Sharma S; Hoener zu Bentrup K; McKinney J; Russell D; Jacobs WJ; Sacchettini J, Structure of isocitrate lyase, a persistence factor of Mycobacterium tuberculosis. Nat. Struct. Biol. 2000, 7 (8), 663-668. [PubMed: 10932251]

(2). Baillie TA, Targeted Covalent Inhibitors for Drug Design. Angew. Chem. Int. Ed. Engl. 2016, 55 (43), 13408-13421. [PubMed: 27539547] 
(3). Singh J; Petter RC; Baillie TA; Whitty A, The resurgence of covalent drugs. Nat Rev Drug Discov 2011, 10 (4), 307-317. [PubMed: 21455239]

(4). Bhusal RP; Bashiri G; Kwai BXC; Sperry J; Leung IKH, Targeting isocitrate lyase for the treatment of latent tuberculosis. Drug Discov Today 2017, 22 (7), 1008-1016. [PubMed: 28458043]

(5). Schloss JV; Cleland WW, Inhibition of isocitrate lyase by 3-nitropropionate, a reactionintermediate analogue. Biochemistry 1982, 21 (18), 4420-4427. [PubMed: 7126549]

(6). Moynihan MM; Murkin AS, Cysteine is the general base that serves in catalysis by isocitrate lyase and in mechanismbased inhibition by 3-nitropropionate. Biochemistry 2014, 53 (1), 178187.

(7). Porter DJ; Bright HJ, 3-Carbanionic substrate analogues bind very tightly to fumarase and aspartase. J. Biol. Chem. 1980, 255 (10), 4772-4780. [PubMed: 7372610]

(8). Gould TA; de Langemheen HV; Munoz-Elias EJ; McKinney JD; Sacchettini JC, Dual role of isocitrate lyase 1 in the glyoxylate and methylcitrate cycles in Mycobacterium tuberculosis. Mol. Microbiol. 2006, 61 (4), 940-947. [PubMed: 16879647]

(9). Pham TV; Murkin AS; Moynihan MM; Harris L; Tyler PC; Shetty N; Sacchettini JC; Huang HL; Meek TD, Mechanism-based inactivator of isocitrate lyases 1 and 2 from Mycobacterium tuberculosis. Proc. Natl. Acad. Sci. USA 2017, 114 (29), 7617-7622. [PubMed: 28679637]

(10). Hwu JR; Tsay SC, Counterattack Reagents - Thiosilanes in the Conversion of Nitro-Compounds to Thiohydroxamic Acids and Thiohydroximates. Tetrahedron 1990, 46 (21), 7413-7428.

(11). Coles CJ; Edmondson DE; Singer TP, Inactivation of succinate dehydrogenase by 3 nitropropionate. J. Biol. Chem. 1979, 254 (12), 5161-5167. [PubMed: 447637]

(12). Ju KS; Gao J; Doroghazi JR; Wang KK; Thibodeaux CJ; Li S; Metzger E; Fudala J; Su J; Zhang JK; Lee J; Cioni JP; Evans BS; Hirota R; Labeda DP; van der Donk WA; Metcalf WW, Discovery of phosphonic acid natural products by mining the genomes of 10,000 actinomycetes. Proc. Natl. Acad. Sci. U. S. A. 2015, 112 (39), 12175-12180. [PubMed: 26324907]

(13). Halkier BA; Gershenzon J, Biology and biochemistry of glucosinolates. Annu. Rev. Plant Biol. 2006, 57, 303-333. [PubMed: 16669764]

(14). Hansen CH; Du L; Naur P; Olsen CE; Axelsen KB; Hick AJ; Pickett JA; Halkier BA, CYP83b1 is the oximemetabolizing enzyme in the glucosinolate pathway in Arabidopsis. J. Biol. Chem. 2001, 276 (27), 24790-24796. [PubMed: 11333274]

(15). Patterson S; Wyllie S, Nitro drugs for the treatment of trypanosomatid diseases: past, present, and future prospects. Trends Parasitol 2014, 30 (6), 289-298. [PubMed: 24776300] 

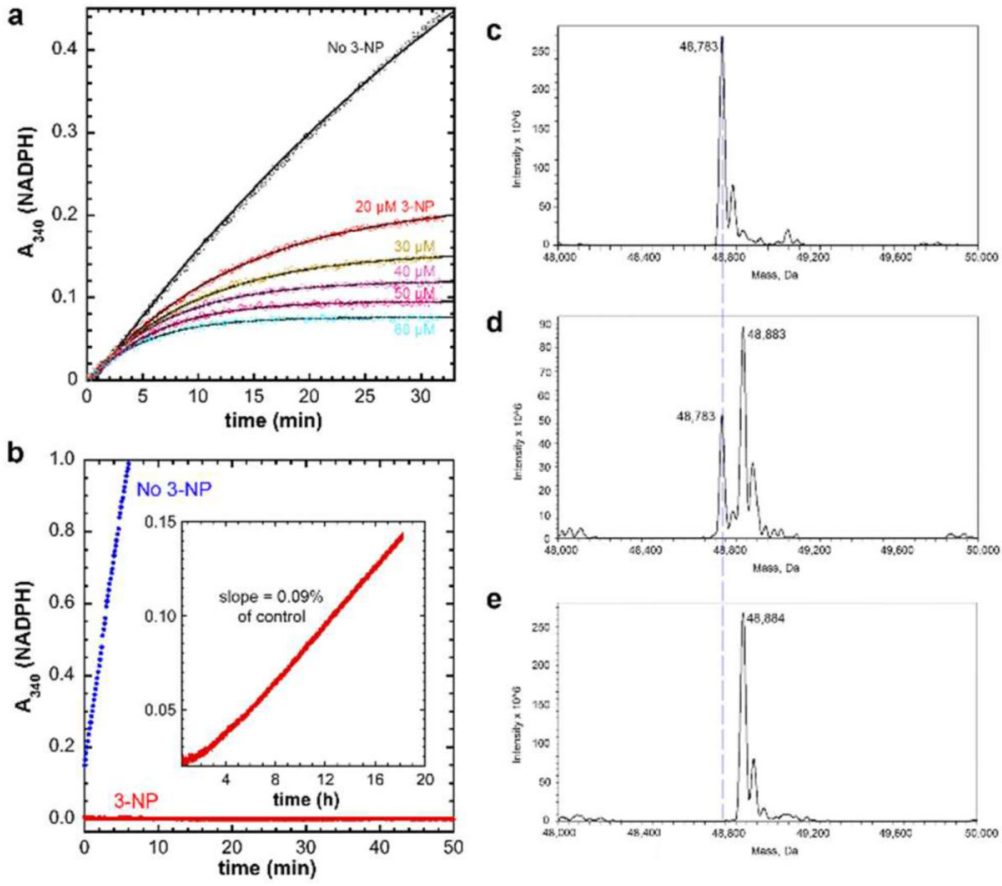

Figure 1.

Kinetic and mass characterization of ICL inhibition by 3-NP. (a) Isocitrate dehydrogenasecoupled assay of ICL activity at $\mathrm{pH} 7.5$ in the presence of 20-60 $\mu \mathrm{M} 3-\mathrm{NP}$. Curves are leastsquares fits to an exponential equation (see SI). (b) Recovery of ICL activity upon 3000-fold dilution after $1 \mathrm{~h}$ preincubation of $0.5 \mathrm{mM}$ ICL with $1 \mathrm{mM}$ glyoxylate and either $1 \mathrm{mM} 3$ NP (red) or no inhibitor (blue). Inset: expansion of the red curve over an extended period. (c)-(e) Deconvoluted ESIMS spectra of ICL after 8 min of incubation (c) alone, (d) with 3NP, or (e) with 3-NP and glyoxylate. The dashed line is centered on the mass of unmodified ICL. 
a

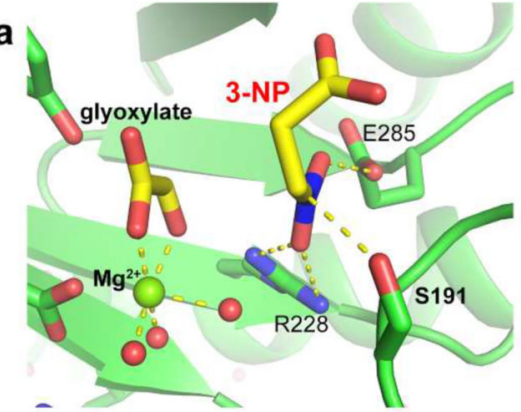

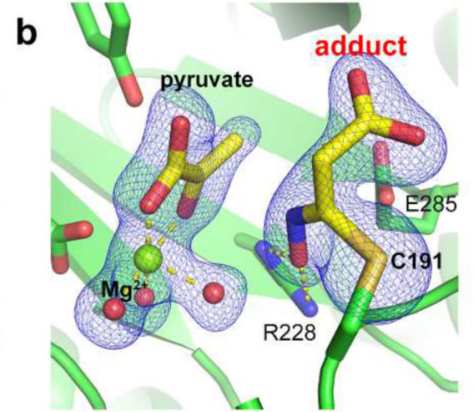

Figure 2.

ICL-3-NP structures. (a) C191S $\bullet \mathrm{Mg}^{2+} \bullet$ glyoxylate $3 \mathrm{NP}$ from PDB $1 \mathrm{~F} 8 \mathrm{I1} .^{1}$ (b) Simulated annealing omit map countered at $3.0 \sigma$ for the thiohydroximate adduct obtained from wildtype ICL, $\mathrm{Mg}^{2+}$, and 3-NP (PDB 6C4A). 
a

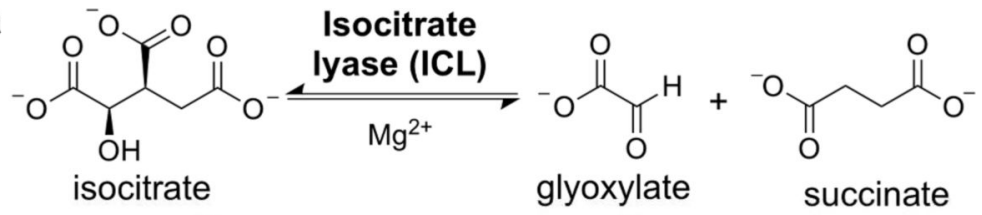

b glyoxylate succinate

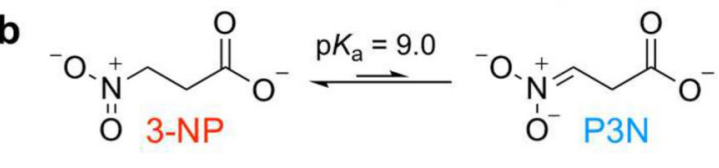

C $E \frac{k_{1}[\mathrm{P} 3 \mathrm{~N}]}{k_{-1}} \mathrm{E} \cdot \mathrm{P} 3 \mathrm{~N} \frac{k_{2}}{k_{-2}}-E^{*} \cdot \mathrm{P} 3 \mathrm{~N}$

d $\mathrm{E} \frac{k_{1}[3-\mathrm{NP}]}{k_{-1}} \mathrm{E} \cdot 3-\mathrm{NP} \stackrel{k_{\text {inact }}}{\longrightarrow} \mathrm{E}-\mathrm{I}$

Scheme 1.

ICL reaction and inhibition by $3-\mathrm{NP}$. 

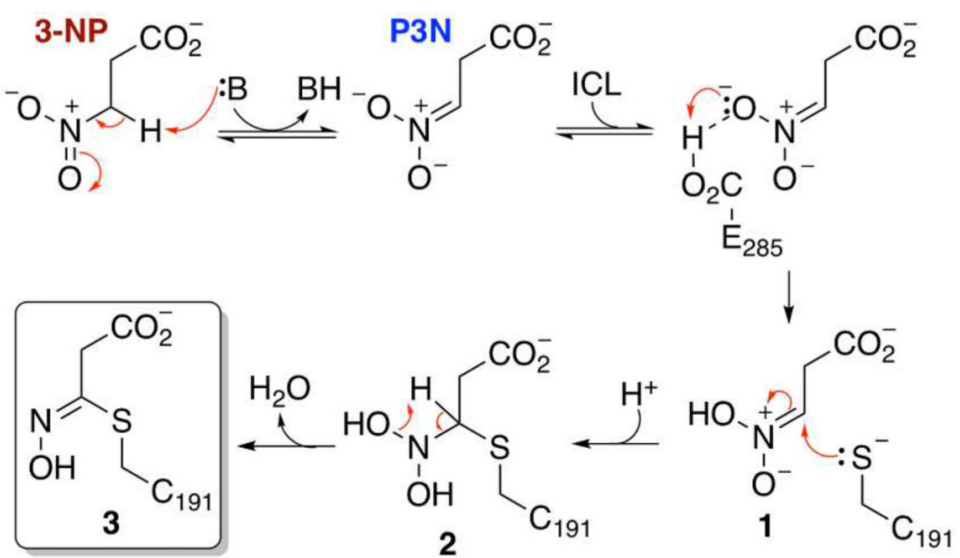

\footnotetext{
${ }^{a} \mathrm{~B}=$ buffer.
}

Scheme 2.

Proposed Mechanism for Thiohydrox mate Formation ${ }^{\mathrm{a}}$ 